\title{
Contact area between a viscoelastic solid and a hard, randomly rough, substrate
}

\author{
B. N. J. Persson a) \\ IFF, FZ-Jülich, 52425 Jülich, Germany \\ O. Albohr \\ Pirelli Reifenwerke, 64733 Höchts/Odenwald, Postfach 1120, Germany \\ C. Creton \\ Laboratoire PCSM, ESPCI-10, rue Vauquelin-75231 Paris Cedex 05, France \\ V. Peveri \\ Pirelli Tire Section, Pirelli Pneumatici S.P.A., Viale Sarca 222, 20126 Milan, Italy
}

(Received 19 December 2003; accepted 17 February 2004)

\begin{abstract}
We study the time-dependent contact area as a viscoelastic solid is squeezed against a randomly rough substrate. Using a recently developed contact mechanics theory we study how the contact area depends on time and on the magnification $\zeta$. Numerical results are presented for self-affine fractal surfaces, and applications to tack, rubber friction, and sealing are given. (C) 2004 American
\end{abstract} Institute of Physics. [DOI: 10.1063/1.1697376]

\section{INTRODUCTION}

The contact between a viscoelastic solid and hard, randomly rough, substrates is a topic of great practical importance, e.g., for pressure sensitive adhesives, rubber friction, and rubber sealing. When a viscoelastic solid is squeezed with a constant force against a rough substrate, the area of real contact will increase monotonically with the contact time. Since rubberlike materials have a wide distribution of relaxation times, the area of real contact will usually increase over a very long time period (which, e.g., could be a year or more). Since the pull-off force depend on the area of real contact, contact theories for viscoelastic solids are important for estimating how the pull-off force (or tack) depend on the applied squeezing pressure and the squeezing time.

Rubberlike materials have elastic modulus $E(\omega)$ that depend strongly on frequency $\omega$. Thus, at very low frequencies they behave as very soft "rubbery" materials, with typical elastic modulus in the range 0.01-1 MPa. At high frequencies they instead behave as hard glassy materials with the elastic modulus of order $1 \mathrm{GPa}$ or more. Thus, as a function of frequency the elastic modulus may increase by a factor of 1000 or more. The transition from the rubbery region to the glassy region is very wide, usually extending over more than three frequency decades. In a contact experiment, the inverse of the contact time is a characteristic frequency; thus for long contact time rubber behave as a "soft" solid and the contact area is "large," while for short contact times it is relatively "hard" and the contact area "small."

The viscoelastic modulus of rubberlike materials is strongly (exponentially or faster) dependent on the temperature. Thus, at high enough temperature most of the rubber relaxation times will be shorter than typical contact times, resulting in a "large" contact area, which is weakly depen-

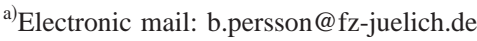

dent on the contact time. At very low temperatures rubber is in a glassy state, characterized by very long relaxation times. In this case the contact area is "small," and very little relaxation occur on the time scale of a typical contact experiment; thus the contact area will increase very slowly with increasing contact time. For intermediate temperatures, where the characteristic frequency $\omega=1 / T$ (where $T$ is the contact time) is somewhere in the transition region between the rubbery region and the glassy region of the viscoelastic modulus $E(\omega)$, the contact area will depend strongly on the contact time $T$.

In a linear viscoelastic material the stress, $\sigma(\mathbf{x}, t)$, is related to the strain $\epsilon(\mathbf{x}, t)$ via

$$
\sigma(\mathbf{x}, t)=\int_{-\infty}^{t} d t^{\prime} E\left(t-t^{\prime}\right) \frac{\partial \epsilon}{\partial t^{\prime}}\left(\mathbf{x}, t^{\prime}\right) .
$$

If we define the Fourier transform

$$
\widetilde{f}(\omega)=\frac{1}{2 \pi} \int_{-\infty}^{\infty} d t f(t) e^{i \omega t},
$$

then

$$
\widetilde{\sigma}(\mathbf{x}, \omega)=\hat{E}(\omega)(-i \omega) \widetilde{\epsilon}(\mathbf{x}, \omega),
$$

where

$$
\hat{E}(\omega)=\int_{0}^{\infty} d t E(t) e^{i \omega t}
$$

It is also convenient to define the frequency-dependent elastic modulus $E(\omega)$ via

$$
E(\omega)=-i \omega \hat{E}(\omega)
$$


Most rheological experiments measure $E(\omega)$ directly, rather than $\hat{E}(\omega)$. $E(t)$ can be obtained from relaxation test experiments, or calculated from the complex frequency-dependent elastic modulus $E(\omega)$.

Suppose that a viscoelastic solid is brought in contact with a hard rough substrate at time $t=0$. That is, the applied (squeezing) force vanish for negative time (no contact), while it is nonzero for $t>0$. In the simplest approach, the contact area is calculated using the standard Greenwood-Williamson $^{1}$ contact mechanics theory, with the modification $^{2}$ that the time-dependent viscoelastic relaxation modulus $E(t)$ is use instead of the constant elastic modulus as in the original theory. However, this approach overlooks the following important effect: Assume that the solids are squeezed together with a constant force at time $t=0$. For very short time the contact will be determined by the short time (or high-frequency) viscoelastic modulus, and the contact area will be "small." Now, as time increases the elastic modulus $E(t)$ decreases and the contact area increases as a result of the growth of the contact "islands" that was formed at time $t=0$, and as a result of the formation of new contact islands for $t>0$ (see Fig. 1). But a volume element of the solid that is deformed at time $t=t_{0}>0$, will give rise to a stress that evolve with time as $\sim E\left(t-t_{0}\right)\left(t>t_{0}\right)$. Thus, the stress distribution at the interface at time $t>0$ will depend on $E\left(t-t^{\prime}\right)$ for all times $0<t^{\prime}<t$, and not just on $E(t)$, as assumed in Ref. 2. This fact has already been pointed out in Ref. 3.

In this paper we present a study of the time dependence of the contact area when a viscoelastic solid is squeezed against a hard, randomly rough, substrate. We present numerical results based on the viscoelastic modulus measured for soft tacky rubber and tire rubber, in contact with hard substrates that have self-affine fractal surface roughness. As illustrations, we discuss the implications for tack, rubber friction, and sealing. We emphasize that the present theory is only valid within the assumption of linear viscoelasticity. Thus, for example, if a polymer in the glassy state is squeezed against a hard rough substrate, local plastic flow (nonlinear process) will occur in the asperity contact regions, and the time dependence of the contact area cannot be predicted by the model presented in this paper. Similarly, for polymer systems that undergo large-amplitude (liquidlike) flow when exposed to external forces for a long time period, will not be accurately described by the present theory. This may be the case for uncross-linked (or weakly cross-linked) polymer systems with low glass transition temperatures, or cross-linked polymer systems with mobile (liquidlike) additives.

\section{CONTACT MECHANICS FOR ELASTIC SOLIDS- A BRIEF REVIEW}

The discussion in this paper will be based on two contact theories, namely the Greenwood-Williamson (GW) theory, ${ }^{1,4}$ and a very recently developed theory. ${ }^{5-7}$ We will use the GW theory in its simplest form where all the surface asperities are approximated by spherical bumps of equal radius. (a)

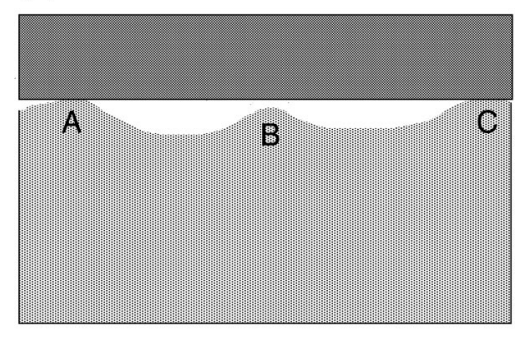

(b)

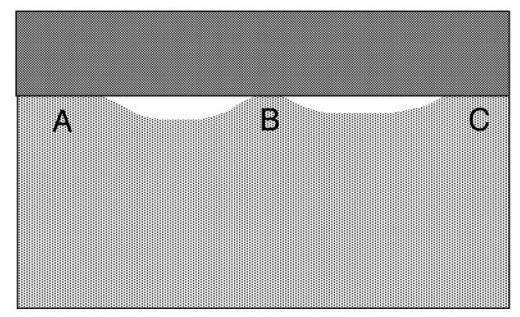

FIG. 1. With increasing time, the contact area between the two solids increases by the growth of existing asperity contact areas (A and C), and by the formation of new asperity contact areas (B).

The theory developed in Ref. 6 is based on the assumption of roughness on many different length scales. The basic idea behind this contact theory is that it is very important not to a priori exclude any roughness length scale from the analysis. Thus, if $A(\lambda)$ is the (apparent) area of contact when the interface is studied at a spatial resolution corresponding to the wavelength $\lambda$, then we study the function $P(\zeta)$ $=A(\lambda) / A(L)$, which is the relative fraction of the surface area where contact occurs on the length scale $\lambda=L / \zeta$. Here $A(L)=A_{0}$ denotes the macroscopic contact area $[L$ is the diameter of the macroscopic contact area so that $A(L)$ $\approx L^{2}$ ]. The function $P(\zeta)$ can be obtained from the interfacial stress distribution $P(\sigma, \zeta)$ at the magnification $\zeta$, via ${ }^{6}$

$$
P(\zeta)=\int_{0}^{\infty} d \sigma P(\sigma, \zeta) .
$$

For a purely elastic solid, $P(\sigma, \zeta)$ satisfy the diffusionlike equation: 6

$$
\frac{\partial P}{\partial \zeta}=f(\zeta) \frac{\partial^{2} P}{\partial \sigma^{2}},
$$

where

$$
f(\zeta)=\frac{\pi}{4}\left(E^{*}\right)^{2} q_{L} q^{3} C(q),
$$

where $q=\zeta q_{L}$ (with $q_{L}=2 \pi / L$ ) and where $E^{*}=E$ / $\left(1-\nu^{2}\right)$. The surface roughness power spectra $C(q)$ is defined by

$$
C(q)=\frac{1}{(2 \pi)^{2}} \int d^{2} x\langle h(\mathbf{x}) h(\mathbf{0})\rangle e^{-i \mathbf{q} \cdot \mathbf{x}},
$$

where $h(\mathbf{x})$ is the height of the rough surface measured from a flat reference plane chosen so that $\langle h\rangle=0$. 


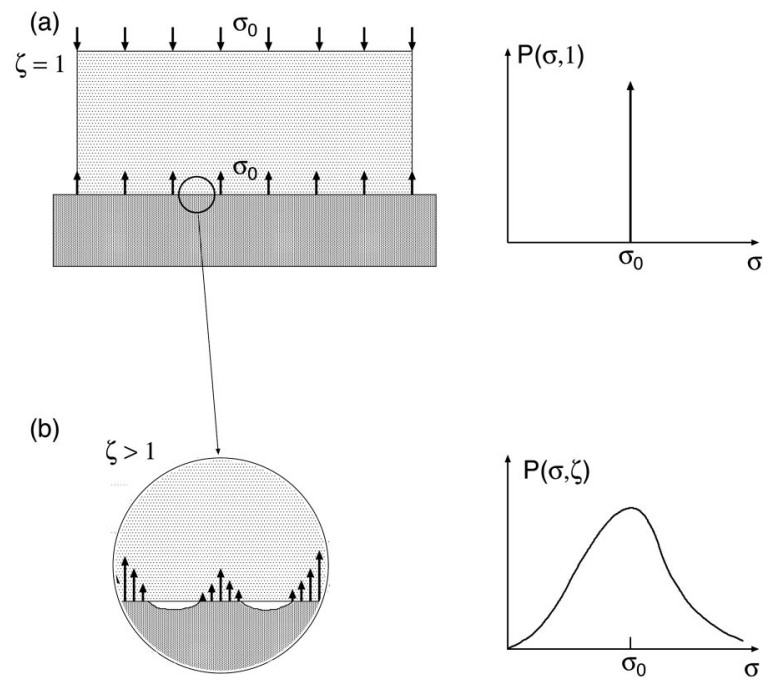

FIG. 2. A rigid block squeezed against an elastic, randomly rough, substrate. The system is studied at different magnifications. At the lowest magnification $\zeta=1$, no surface roughness can be observed and the block makes apparent contact with the substrate everywhere, resulting in a stress distribution in the form of a Dirac delta function centered at the applied stress $\sigma_{0}$. When the system is studied at increasing magnification $\zeta>1$, surface roughness can be observed, resulting in a broadened stress distribution (see the text for details).

The physical meaning of Eq. (2) is as follows: When the system is studied at the lowest magnification $\zeta=1$, no surface roughness can be observed and the block makes (apparent) contact with the substrate everywhere in the nominal contact area. In this case, if we neglect friction at the interface, the stress at the interface will everywhere equal the applied stress $\sigma_{0}$, see Fig. 2(a), so that $P(\sigma, 1)=\delta(\sigma$ $\left.-\sigma_{0}\right)$. When we increase the magnification, we observe surface roughness with wavelength down to $\lambda=L / \zeta$. In this case one may observe some noncontact regions, as shown in Fig. 2(b). Since the stress must go continuously to zero at the edges of the boundary between the contact and noncontact regions, it follows that the stress distribution $P(\sigma, \zeta)$ will have a tail extending the whole way down to the zero stress, as indicated in Fig. 2(b) (right). There will also be a tail toward larger stresses $\sigma>\sigma_{0}$ because the average stress must be equal to $\sigma_{0}$. Thus, with increasing magnification, the stress distribution will broaden (without limit), as indicated in Fig. 2 (right). This is similar to the broadening by diffusion of a system of particles initially localized to one point $x_{0}$ in space.

Using (1) and (2) gives

$$
P(\zeta)=\frac{2}{\pi} \int_{0}^{\infty} d x \frac{\sin x}{x} e^{-x^{2} G(\zeta)}=\operatorname{erf}\left(\frac{1}{2 \sqrt{ } G}\right),
$$

where

$$
G(\zeta)=\int_{0}^{q_{L} \zeta} d \zeta^{\prime} \frac{f\left(\zeta^{\prime}\right)}{\sigma_{0}^{2}}=\frac{\pi}{4}\left(\frac{E^{*}}{\sigma_{0}}\right)^{2} \int_{0}^{q_{L} \zeta} d q q^{3} C(q) .
$$

Note that for small load $\sigma_{0}, G \gg 1$, and in this case (3) reduces to $P(\zeta) \approx P_{1}(\zeta)$, where

$$
P_{1}(\zeta)=[\pi G(\zeta)]^{-1 / 2}
$$

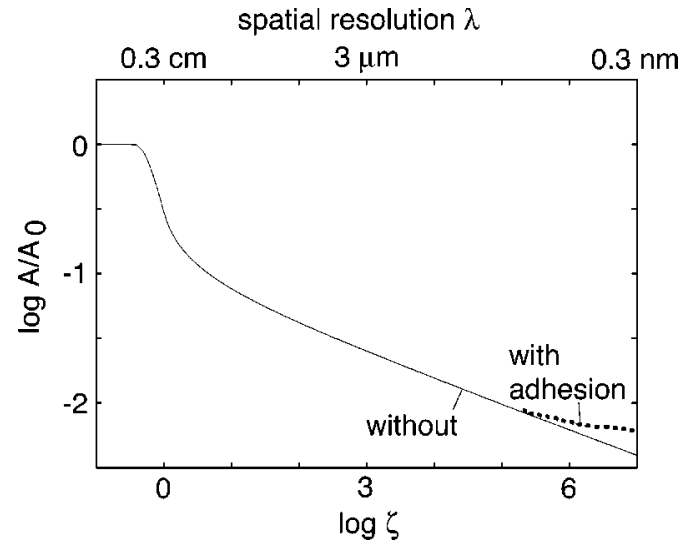

FIG. 3. The logarithm of the normalized contact area, $A(\zeta) / A_{0}$, for a tiretread rubber block in contact with an asphalt road as a function of the logarithm of the magnification, $\zeta$ (lower scale), or the spatial resolution $\lambda$ (upper scale). The squeezing pressure is $0.4 \mathrm{MPa}$ and the contact time $1 \mathrm{~s}$. The solid and dashed lines are without and with the adhesional interaction included in the calculation.

Since $G \sim 1 / \sigma_{0}^{2}$ it follows that the area of real contact is proportional to the load for a small load. Using (3) and (4a) we can write, in a general case,

$$
P(\zeta)=\operatorname{erf}\left(\frac{\sqrt{ } \pi}{2} P_{1}(\zeta)\right)
$$

In this paper we neglect the influence of the attractive adhesional interaction between the solids at the interface. Here we will briefly describe under which conditions this is a good approximation. Assume that an elastic solid with a flat surface is squeezed against a nominally flat substrate. Let $\sigma_{0}$ be the nominal pressure in the nominal contact area. Let us first neglect the adhesional interaction. In this case the contact area between the solids will typically depend on the magnification as shown by the solid line in Fig. 3. When we increase the magnification, the contact area $A(\zeta)$ will continuously decrease while the local pressures in the (apparent) contact areas increases. Let us study the system at the magnification $\zeta=L / \lambda$, corresponding to the spatial resolution $\lambda$. We now take into account the adhesional interaction. In a first approximation the adhesional interaction will give rise to an additional pressure so that the contact area at the magnification $\zeta$ depends on $\sigma_{0}+\sigma_{a}(\zeta)$, where the adhesion pressure, ${ }^{8}$

$$
\sigma_{a}(\zeta)=\left[\frac{\pi \gamma_{\mathrm{eff}}(\zeta) E^{*}}{\lambda}\right]^{1 / 2}
$$

In a typical case, because of surface roughness, the effective interfacial surface energy $\gamma_{\text {eff }}<\gamma$ is smaller than the (bar) interfacial surface energy $\gamma=\gamma_{1}+\gamma_{2}-\gamma_{12}$ observed when two perfectly flat surfaces are brought together. However, as the magnification $\zeta$ increases (i.e., the resolution $\lambda$ approaches an atomic distance), $\gamma_{\text {eff }}$ will approach $\gamma$. If we use $\gamma$ instead of $\gamma_{\text {eff }}$ in (5), we get an overestimation of the importance of the adhesional interaction. As an illustration of the usefulness of the concept of adhesion pressure, in the 
Appendix we present a short derivation of the standard JKR result for the adhesion of an elastic ball to a rigid flat substrate.

In a typical application, e.g., a tire on a road or during the contact phase in a tack experiment, the external squeezing pressure is of order $\sigma_{0} \approx 1 \mathrm{MPa}$. Assuming that $\gamma$ has its origin in the van der Waals interaction (as is almost always the case in practical applications) we have $\gamma \approx 1 \mathrm{meV} / \AA^{2}$. The equation,

$$
\left[\frac{\pi \gamma E^{*}}{\lambda}\right]^{1 / 2} \approx 1 \mathrm{MPa}
$$

gives $\lambda \approx 100 \AA$ if $E^{*}=1 \mathrm{MPa}$, and $\lambda=1 \AA$ if $E^{*}$ $=0.01 \mathrm{MPa}$. Note that at a given squeezing pressure, the length-scale $\lambda$ where adhesion becomes important, decreases with decreasing elastic modulus, and, as indicated above, for very soft elastic solids, e.g. tack, the adhesional interaction can in a typical case (where $\sigma_{0} \sim 1 \mathrm{MPa}$ ) be neglected during the contact formation process. But even for tire rubber the adhesional interaction will become important only when we study the contact area with a spatial resolution of least $\lambda$ $=100 \AA$. This is illustrated in Fig. 3 for a tire tread rubber squeezed against a road surface. Since rubber friction on rough surfaces is mainly due to viscoelastic deformations of the rubber on length scales larger than $1 \mu \mathrm{m}$ (see Refs. 6, 28 ), it is clear that the adhesional interaction will have a small influence on the friction coefficient in this case. However, if we study a property such as the pull-off force that only depend on the area of real contact, then the adhesioninduced enhancement of the contact area may be important in some cases. Note also that even if the area of real contact is determined mainly by the squeezing force, as will be the case in most applications to tack, the adhesional interaction in the area of real contact will be the sole origin of the pull-off force. However, the exact origin of the pull-off force is a much more complicated problem than the contact formation process, in general, involving cavity formation, stringing, and other nonlinear processes, and cannot be successfully studied without a detailed knowledge of the linear and nonlinear mechanical properties of the rubber itself. ${ }^{9-11}$

Note that the adhesional interaction becomes more important when the nominal contact pressure $\sigma_{0}$ decreases. Thus, adhesion is important when $\lambda<\lambda_{a}$, where $\lambda_{a}$ $=\gamma E^{*} / \sigma_{0}^{2}$ increases with decreasing $\sigma_{0}$. This effect may be important for formula 1 tires (which are very wide), for which, in contrast to normal personal car tires, it is believed that adhesion is important because of the special design of the tread tire rubber (which contains sticky substances).

\section{GREENWOOD-WILLIAMSON (GW) CONTACT THEORY FOR VISCOELASTIC SOLIDS}

In the simplest description of a rough surface, the surface asperities are all considered as spherical cups with equal radius $R$ but random heights. Random surfaces have Gaussian height distribution. However, for a Gaussian height distribution the GW model does not give a contact area $A$ that is linearly dependent on the squeezing force $F_{N}$, as observed in more realistic treatments as long as the contact area is

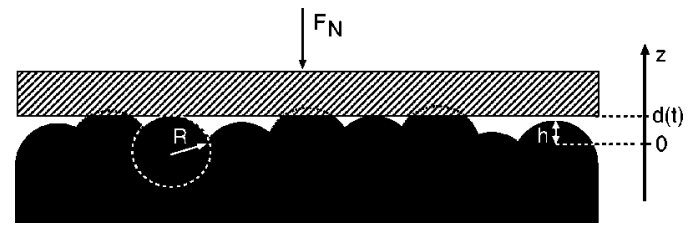

FIG. 4. Contact between a rough hard substrate and an elastic solid. All asperities with height $h$ greater than $d(t)$ makes contact.

small compared to the nominal surface area. However, if the height distribution is assumed to be exponential, $P(h)$ $=B \exp (-h / \lambda)$, then $A$ will depend linearly on $F_{N}$, and we will therefore focus on this limiting case in the present section.

We will need the following results obtained for a hard spherical asperity squeezed against the flat surface of a viscoelastic solid. The contact area will be circular with a radius $r(t)$ and area $a=\pi r^{2}$, related to the penetration $\delta(t)$ (the indentation depth into the elastic solid) via the standard Hertz formulas, ${ }^{12}$

$$
\begin{aligned}
& \delta(t)=r^{2}(t) / R, \\
& a(t)=\pi R \delta(t) .
\end{aligned}
$$

The normal (squeezing) force $f_{N}$ is related to the radius $r(t)$ via

$$
f_{N}(t)=\frac{8}{3 R} \int_{-\infty}^{t} d t^{\prime} E^{*}\left(t-t^{\prime}\right) \frac{d}{d t^{\prime}} r^{3}\left(t^{\prime}\right),
$$

where $E^{*}=E /\left(1-\nu^{2}\right)$. These results are only valid as long as the loading history is such that the contact area increases with time, and we will only focus on this case in what follows.

Assume that the elastic solid is located at $z=d(t)$ above the rough substrate; see Fig. 4. If there are $N_{0}$ spherical asperities, then the number of asperities in contact with the elastic wall will be

$$
N(t)=\int_{d(t)}^{\infty} d h N_{0} P(h) .
$$

Since $\delta(t)=h-d(t)$, using (7) we get the area of real contact,

$$
A(t)=\int_{d(t)}^{\infty} d h N_{0} P(h) \pi R[h-d(t)] .
$$

Using (8), the total normal force $F_{N}$ is given by

$$
\begin{gathered}
F_{N}(t)=\frac{8 \sqrt{ } R}{3} \int_{d(t)}^{\infty} d h N_{0} P(h) \int_{-\infty}^{t} d t^{\prime} E^{*}\left(t-t^{\prime}\right) \\
\times \theta\left[h-d\left(t^{\prime}\right)\right] \frac{d}{d t^{\prime}}\left[h-d\left(t^{\prime}\right)\right]^{3 / 2} .
\end{gathered}
$$

Let us assume that the squeezing is turned on at time $t=0$ so that $F_{N}=0$ for $t<0$ and $F_{N}=F_{0}=$ const $>0$ for $t>0$. In this case we get from (11) for $t>0$, 


$$
\begin{aligned}
F_{0}= & F_{0} \frac{E^{*}(t)}{E^{*}(0)}+\frac{8 \sqrt{ } R}{3} \int_{d(t)}^{\infty} d h N_{0} P(h) \\
& \times \int_{0}^{t} d t^{\prime} E^{*}\left(t-t^{\prime}\right) \theta\left[h-d\left(t^{\prime}\right)\right] \frac{d}{d t^{\prime}}\left[h-d\left(t^{\prime}\right)\right]^{3 / 2} .
\end{aligned}
$$

Let us introduce

$$
v(t)=-\dot{d}(t),
$$

which is the relative velocity between the surfaces. Thus, (12) can be written as

$$
\begin{aligned}
F_{0}= & F_{0} \frac{E^{*}(t)}{E^{*}(0)}+4{ }_{\sqrt{ }} R \int_{d(t)}^{\infty} d h N_{0} P(h) \\
& \times \int_{0}^{t} d t^{\prime} E^{*}\left(t-t^{\prime}\right) \theta\left[h-d\left(t^{\prime}\right)\right] v\left(t^{\prime}\right)\left[h-d\left(t^{\prime}\right)\right]^{1 / 2} .
\end{aligned}
$$

Substituting

$$
P(h)=B e^{-h / \lambda}
$$

in (9), (10), and (12) and performing the $h$ integrals gives

$$
\begin{aligned}
& N(t)=N_{0} B \lambda e^{-d(t) / \lambda}, \\
& A(t)=N_{0} B \pi R \lambda^{2} e^{-d(t) / \lambda}, \\
& F_{0}=F_{0} \frac{E^{*}(t)}{E^{*}(0)}+\alpha \int_{0}^{t} d t^{\prime} E^{*}\left(t-t^{\prime}\right) v\left(t^{\prime}\right) e^{-d\left(t^{\prime}\right) / \lambda}
\end{aligned}
$$

where

$$
\alpha=2(\pi R)^{1 / 2} \lambda^{3 / 2} N_{0} B .
$$

Equation (16) is an integral equation for $d(t)$, which can be solved as follows. Let us assume that

$$
E^{*}(t)=E_{0}+\left(E_{\infty}-E_{0}\right) e^{-t / \tau^{*}},
$$

so that

$$
\dot{E}^{*}(t)=\left(E_{\infty}-E_{0}\right)\left(-1 / \tau^{*}\right) e^{-t / \tau^{*}}
$$

and

$$
\ddot{E}^{*}(t)=-\dot{E}^{*}(t) / \tau^{*}
$$

The time derivative of (16) gives

$$
\begin{aligned}
0= & F_{0} \frac{\dot{E}^{*}(t)}{E^{*}(0)}+\alpha E^{*}(0) v(t) e^{-d(t) / \lambda} \\
& +\alpha \int_{0}^{t} d t^{\prime} \dot{E}^{*}\left(t-t^{\prime}\right) v\left(t^{\prime}\right) e^{-d\left(t^{\prime}\right) / \lambda}
\end{aligned}
$$

or

$$
\begin{aligned}
v(t)= & -\frac{F_{0}}{\alpha E^{*}(0)} \frac{\dot{E}^{*}(t)}{E^{*}(0)} e^{d(t) / \lambda} \\
& -\int_{0}^{t} d t^{\prime} \frac{\dot{E}^{*}\left(t-t^{\prime}\right)}{E^{*}(0)} v\left(t^{\prime}\right) e^{\left[d(t)-d\left(t^{\prime}\right)\right] / \lambda}
\end{aligned}
$$

Taking the time derivative of this equation and using (18) and (19) gives, after some simplifications,

$$
\dot{v}(t)=-\frac{v(t)}{\tau}-\frac{v^{2}(t)}{\lambda},
$$

where $\tau=\left(E_{\infty} / E_{0}\right) \tau^{*}$. From (20) we get the boundary condition

$$
v(0)=-\frac{\dot{E}^{*}(0)}{E^{*}(0)} \frac{F_{0}}{\alpha E^{*}(0)} e^{d(0) / \lambda} .
$$

Equation (21) is easy to integrate to get

$$
v(t)=\frac{\lambda / \tau}{C e^{t / \tau}-1},
$$

where $C$ is a constant. Next, using that $v=-\dot{d}(t)$, we get

$$
d(t)=d_{0}+\frac{1}{\lambda} \ln \left(C-e^{-t / \tau}\right) .
$$

Now, in the limit $t \rightarrow 0$ the contact mechanics is determined by the high-frequency elastic modulus $E_{\infty}$ so that it is easy to calculate

$$
F_{0}=\alpha E_{\infty} \lambda e^{-d(0) / \lambda} .
$$

Using (22) and (25) gives

$$
v(0)=\frac{\lambda}{\tau^{*}}\left(1-\frac{E_{0}}{E_{\infty}}\right) \text {. }
$$

But from (23),

$$
v(0)=\frac{\lambda / \tau}{C-1},
$$

and combining this with (26) gives

$$
C=\frac{E_{\infty}}{E_{\infty}-E_{0}} .
$$

Using (24), (27), and (15) gives

$$
A(t)=A_{1}\left[1+\left(\frac{E_{0}}{E_{\infty}}-1\right) e^{-t / \tau}\right],
$$

where $A_{1}$ is the contact area for large time $t \rightarrow \infty$. Note that the short-time contact area $A(0)=A_{1} E_{0} / E_{\infty} \ll A_{1}$ since typically $E_{0} / E_{\infty} \approx 10^{-3}$.

\section{PHENOMENOLOGICAL VISCOELASTIC CONTACT THEORY}

Consider an elastic solid with a flat smooth surface squeezed against a randomly rough substrate. Contact mechanics theories $^{6,13}$ show that as long as the area of real contact, $A$, is small compared to the nominal contact area $A_{0}$, the area of real contact is proportional to the normal (squeezing) force $F_{N}$. Thus, the theory developed in Ref. 6, and briefly described in Sec. II, gives

$$
A(\zeta)=\frac{2}{\pi Q(\zeta)} \frac{F_{N}}{E^{*}}
$$

where $Q(\zeta)$ depends on the surface roughness power spectra $C(q)$ : 


$$
Q=\left(\int_{0}^{q_{L} \zeta} d q q^{3} C(q)\right)^{1 / 2} .
$$

Equation (29) can also be written as

$$
E^{*} P(\zeta)=\frac{2 \sigma_{0}}{\pi Q(\zeta)}
$$

where $\sigma_{0}=F_{N} / A_{0}$ is the nominal normal (squeezing) stress, and $P(\zeta)=A(\zeta) / A_{0}$ the relative contact area at the magnification $\zeta$. If the stress $\sigma_{0}(t)$ depend on time, the area of contact $A(\zeta, t)$ will also depend on time and is given by (31).

The results above was derived for a purely elastic solid. We now generalize the theory to a viscoelastic solid, characterized by the viscoelastic modulus $E^{*}(t)$. We tentatively replace (31) with

$$
\int_{-\infty}^{t} d t^{\prime} E^{*}\left(t-t^{\prime}\right) \frac{\partial P\left(\zeta, t^{\prime}\right)}{\partial t^{\prime}}=\frac{2 \sigma_{0}(t)}{\pi Q(\zeta)} .
$$

Let us define the Fourier transform,

$$
\widetilde{\sigma}_{0}(\omega)=\frac{1}{2 \pi} \int_{-\infty}^{\infty} d t \sigma_{0}(t) e^{i \omega t},
$$

and the inverse transform

$$
\sigma_{0}(t)=\int_{-\infty}^{\infty} d \omega \widetilde{\sigma}_{0}(\omega) e^{-i \omega t} .
$$

If we assume that $\sigma_{0}(t)$ vanishes for $t= \pm \infty$ we get from (32),

$$
\hat{E}^{*}(\omega)(-i \omega t) \widetilde{P}(\zeta, \omega)=\frac{2 \widetilde{\sigma}_{0}(t)}{\pi Q(\zeta)},
$$

where we have defined

$$
\hat{E}^{*}(\omega)=\int_{0}^{\infty} d t E^{*}(t) e^{i \omega t} .
$$

Using (33), we get

$$
\widetilde{P}(\zeta, \omega)=\frac{2}{\pi Q(\zeta)} \frac{\widetilde{\sigma}_{0}(\omega)}{\hat{E}^{*}(\omega)(-i \omega)}=\frac{2}{\pi Q(\zeta)} \frac{\widetilde{\sigma}_{0}(\omega)}{E^{*}(\omega)}
$$

and

$$
P(\zeta, t)=\frac{2}{\pi Q(\zeta)} \int_{-\infty}^{\infty} d \omega \frac{\widetilde{\sigma}_{0}(\omega)}{E(\omega)} e^{-i \omega t} .
$$

Now, assume that

$$
E^{*}(t)=E_{0}+\left(E_{\infty}-E_{0}\right) e^{-t / \tau^{*}} .
$$

In this case,

$$
\hat{E}^{*}(\omega)=\frac{E_{0}}{0^{+}-i \omega}+\frac{E_{\infty}-E_{0}}{1 / \tau^{*}-i \omega}
$$

and

$$
\begin{aligned}
E(\omega) & =(-i \omega) \hat{E}(\omega) \\
& =E_{0}+\frac{\left(E_{\infty}-E_{0}\right) \omega}{\omega+i / \tau^{*}}=\frac{i E_{0} / \tau^{*}+E_{\infty} \omega}{\omega+i / \tau^{*}} .
\end{aligned}
$$

Now, let us assume that $\sigma_{0}(t)=\sigma_{0}$ for $0<t<T$ and zero otherwise. In this case,

$$
\widetilde{\sigma}_{0}(\omega)=\frac{\sigma_{0} / 2 \pi}{0^{+}-i \omega}\left(1-e^{i \omega T}\right) .
$$

Substituting (35) and (36) in (34) gives

$$
\begin{aligned}
P(\zeta, t)= & \frac{2}{\pi Q} \frac{\sigma_{0}}{E_{\infty}} \frac{(-1)}{2 \pi i} \\
& \times \int_{-\infty}^{\infty} d \omega \frac{\omega+i / \tau^{*}}{(\omega+i / \tau)\left(\omega+i 0^{+}\right)}\left(1-e^{i \omega T}\right) e^{-i \omega t},
\end{aligned}
$$

where $1 / \tau=\left(E_{0} / E_{\infty}\right) / \tau^{*}$. Performing the integral in (37) gives $P(\zeta, t)=0$ for $t<0$, while for $0<t<T$,

$$
P(\zeta, t)=\frac{2}{\pi Q} \frac{\sigma_{0}}{E_{0}}\left[1+\left(\frac{E_{0}}{E_{\infty}}-1\right) e^{-t / \tau}\right],
$$

which agree with (28). This equation is only valid for such low load that $P(\zeta, t) \ll 1$. However, we may tentatively obtain an expression for all loads as follows: First, comparing (38) with (4) gives

$$
G=\frac{\pi}{4} \int_{0}^{q_{L} \zeta} d q q^{3} C(q)\left(\frac{E_{0}}{\sigma_{0}}\right)^{2}\left[1+\left(\frac{E_{0}}{E_{\infty}}-1\right) e^{-t / \tau}\right]^{-2} .
$$

We then use this expression in the formula (4b) to get

$$
P(\zeta, t)=\operatorname{erf}\left(\frac{1}{2 \sqrt{ } G(\zeta, t)}\right) .
$$

More generally, if

$$
P_{1}(\zeta, t)=\frac{2}{\pi Q(\zeta)} \int_{-\infty}^{\infty} d \omega \frac{\widetilde{\sigma}_{0}(\omega)}{E^{*}(\omega)} e^{-i \omega t}
$$

denote the relative contact area to linear order in $\sigma_{0}$, then in analogy with (4b), we propose to use

$$
P(\zeta, t)=\operatorname{erf}\left(\frac{\sqrt{ } \pi}{2} P_{1}(\zeta, t)\right),
$$

for all values of $\sigma_{0}$. For the applied stress given by Eq. (36), we have

$$
\begin{aligned}
P_{1}(\zeta, t) & =\frac{2 \sigma_{0}}{\pi Q(\zeta)} \frac{1}{2 \pi} \int_{-\infty}^{\infty} d \omega \frac{1-e^{i \omega T}}{-i \omega} \frac{e^{-i \omega t}}{E^{*}(\omega)} \\
& =\frac{2 \sigma_{0}}{\pi^{2} Q(\zeta)} \operatorname{Re} \int_{0}^{\infty} d \omega \frac{1-e^{i \omega T}}{-i \omega} \frac{e^{-i \omega t}}{E^{*}(\omega)} .
\end{aligned}
$$

This result does, of course, not depend on $T$ as long as $t$ $<T$ (causality). Thus, we are free to choose for $T$ any value larger than the time $t$ under consideration. It turns out to be convenient in the numerical evaluation of $P_{1}(\zeta, t)$ to choose $T=a t$ where $a>1$ but of order 1, e.g., 1.5. Equations (40) and (41) have been used in all the following numerical calculations. 


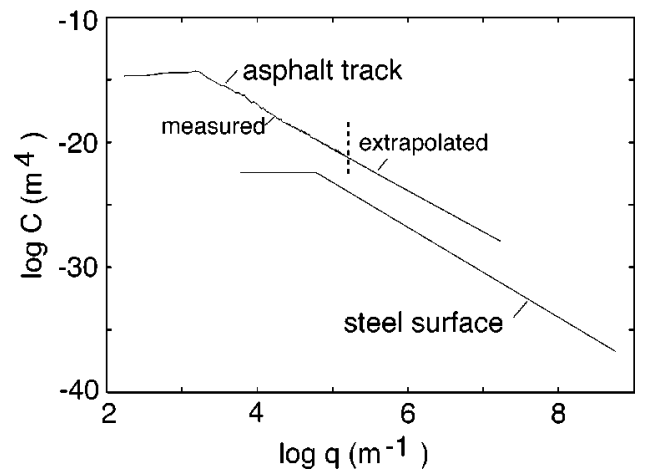

FIG. 5. The logarithm of the power spectra $C(q)$ as a function of the logarithm of the wave vector $q$. Results are shown for an asphalt road track and for a steel surface.

\section{NUMERICAL RESULTS AND APPLICATIONS}

In this section we will present three different applications of the viscoelastic contact theory developed above, namely to (a) tack of pressure-sensitive adhesives (PSA), (b) rubber friction, and (c) sealing. We will not present any detailed comparison of the theory with experiments, but rather just indicate three different applications where viscoelastic contact mechanics should be very important. In the future we plan to present a detailed comparison of the theory with experiments.

We will apply the theory for two different substrates, namely a polished "steel" surface that is assumed to be selfaffine fractal, ${ }^{14}$ with the fractal dimension $D_{f}=2.2$ (corresponding to the Hurst exponent $H=0.8$ ), which is typical for surfaces prepared, e.g., by sandblasting or (for brittle materials) by cleaving. The surface roughness power spectra $C(q)$ is shown in Fig. 5. Note that $C(q)=$ const for $q<q_{0}$ $=6 \times 10^{4} \mathrm{~m}^{-1}$, and the self-affine fractal for $q>q_{0}$. The "steel" surface has the surface root-mean-square (rms) roughness $1 \mu \mathrm{m}$. In the context of tack we also consider surfaces with other rms roughness amplitudes, namely, $h_{0}$ $=0.25,4,5,10,20$, and $100 \mu \mathrm{m}$, but with all the other parameters the same as above; we will refer to these surfaces as "steel" surfaces.

The second surface is an asphalt road track for which we have measured the height profile $h(\mathbf{x})$ using an optical method. From the measured data we have calculated $C(q)$ using a recently developed computer code. ${ }^{15}$ The resulting power spectra is also shown in Fig. 5. In this case $C(q)$ is nearly perfectly self-affine fractal for $q>q_{0}=1694 \mathrm{~m}^{-1}$, the fractal dimension being $D_{f} \approx 2.3$ and the root-mean-square roughness $h_{\mathrm{rms}}=0.26 \mathrm{~mm}$.

For later convenience we define the cut-off or roll-off wavelength $\lambda_{0}=2 \pi / q_{0}$. It is often convenient to refer the magnification $\zeta=1$ to the length scale $\lambda_{0}$, rather than to the linear size $L$ of the rubber block. Thus, if we study the system at the resolution $\lambda$, then $\zeta=\lambda_{0} / \lambda$. In this case it is clear that if we study the system with a resolution $\lambda>\lambda_{0}$ (but, of course, $\lambda<L$ ), then $\zeta<1$. In particular, when the resolution equals the size $L$ of the block (or the nominal contact area), then $\zeta=\lambda_{0} / L$, which is the smallest possible $\zeta$. Some surfaces, e.g., surfaces prepared by fracture, are self-affine frac-
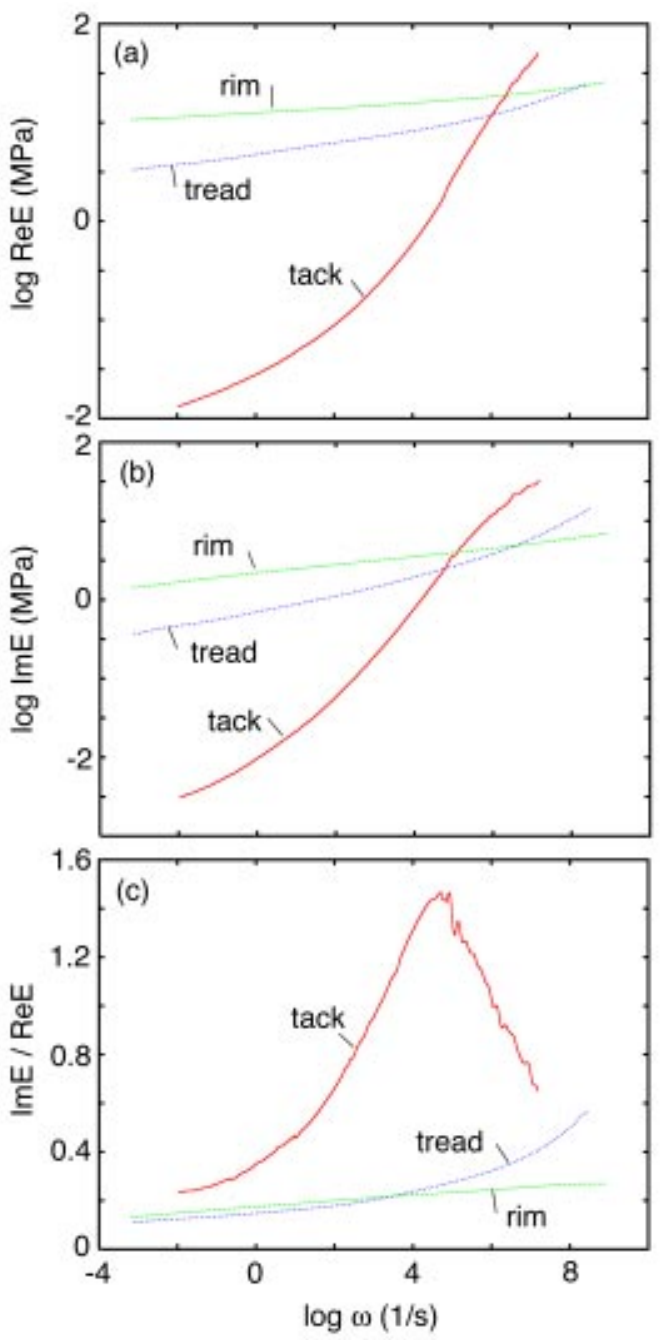

FIG. 6. (a) The real and (b) the imaginary part of the complex viscoelastic modulus $E(\omega)$, and (c) the loss tangent $\operatorname{Im} E / \operatorname{Re} E$ as a function of frequency. Results are shown for a typical PSA rubber, and for a tire rim and tread rubber.

tal the whole way up to the lateral size $L$ of the surface. In these case there is no roll-off wave vector in the $C(q)$ spectra, and the only reasonable definition of the magnification refers to the length scale $L$ so that $\zeta=L / \lambda>1$. In the following the magnification $\zeta=1$ will always refer to the roll-off wavelength $\lambda_{0}$.

The viscoelastic modulus of the PSA rubber, rim rubber, and tread rubber used in our numerical calculations are shown in Fig. 6.

\section{A. Tack}

Pressure sensitive adhesives are used in many important applications, e.g., for Scotch tape, post-it pads, and selfadhesive labels and envelopes. The adhesive consist of a very thin layer (usually of order $\sim 20-100 \mu \mathrm{m}$ ) of a very soft (weakly cross-linked) rubber compound. The low-frequency elastic modulus is typically only $0.01 \mathrm{MPa}$, which is $\sim 100$ times lower than the rubber used for tires; see Fig. 6. As a result of the low elastic modulus, nearly complete contact will occur in the apparent contact area, even for relative low squeezing pressures and large surface roughness. This is in 

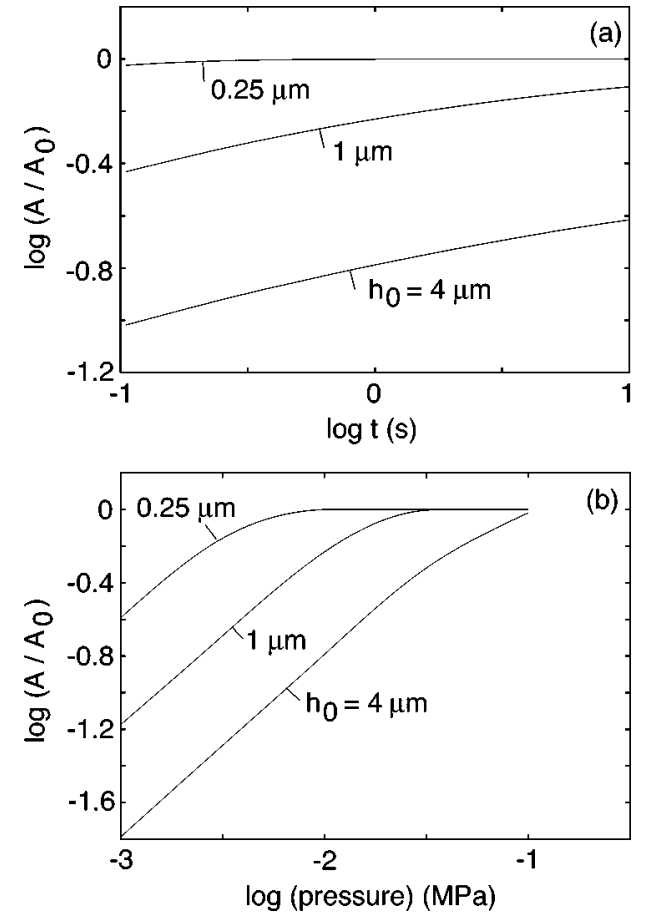

FIG. 7. (a) The logarithm of the relative contact area at the magnification $\zeta=10^{5}$ corresponding to the wavelength $\lambda \approx 1 \mathrm{~nm}$, as a function of the logarithm of the contact time. For the contact pressure $\sigma_{0}=0.01 \mathrm{MPa}$. (b) The logarithm of the relative contact area (after $1 \mathrm{~s}$ of contact) at the magnification $\zeta=10^{5}$, as a function of the logarithm of the contact pressure. For a self-affine fractal substrate surface with the fractal dimension $D_{f}=2.2$, the roll-off wave vector $q_{0}=6 \times 10^{4} \mathrm{~m}^{-1}$ and rms roughness amplitudes $h_{0}$ $=0.25,1$ and $4 \mu \mathrm{m}$. For PEHA-AA at the temperatures $T=20^{\circ} \mathrm{C}$.

contrast to tire rubber that under similar conditions would give a contact area of only a few $\%$ of the nominal contact area (see below). The theory presented above and the numerical results presented below are for a semi-infinite viscoelastic solid and not for a thin confined viscoelastic slab as in most applications to tack. Nevertheless, if the amplitude and the wavelength of the surface roughness are small compared to the thickness of the viscoelastic slab, then the present model should give approximately correct results also for pressure-sensitive adhesives.

We have performed calculations for two different standard rubber tack compounds representative of acrylic PSA used in applications, namely poly(2ethylhexyl acrylate) (PEHA) and PEHA with 2\% acrylic acid (PEHA-AA). The results are summarized later in Figs. 7, 10, 11, 12, and 13.

Figure 7 shows results for PEHA-AA at $T=20^{\circ} \mathrm{C}$, in contact with "steel" surfaces with the rms roughness amplitudes $h_{0}=0.25,1$, and $4 \mu \mathrm{m}$. Figure 7(a) shows the logarithm of the relative contact area for at the magnification $\zeta$ $=10^{5}$ corresponding to the wavelength $\lambda \approx 1 \mathrm{~nm}$, as a function of the logarithm of the contact time, for the contact pressure $\sigma_{0}=0.01 \mathrm{MPa}$. Figure $7(\mathrm{~b})$ shows the logarithm of the relative contact area (after $1 \mathrm{~s}$ of contact), again at the magnification $\zeta=10^{5}$, as a function of the logarithm of the contact pressure.

It is interesting to compare the results in Fig. 7 with the experimental data reported on in Ref. 16 and shown in Fig. 8. The figure shows the dependence of the maximum pull-off
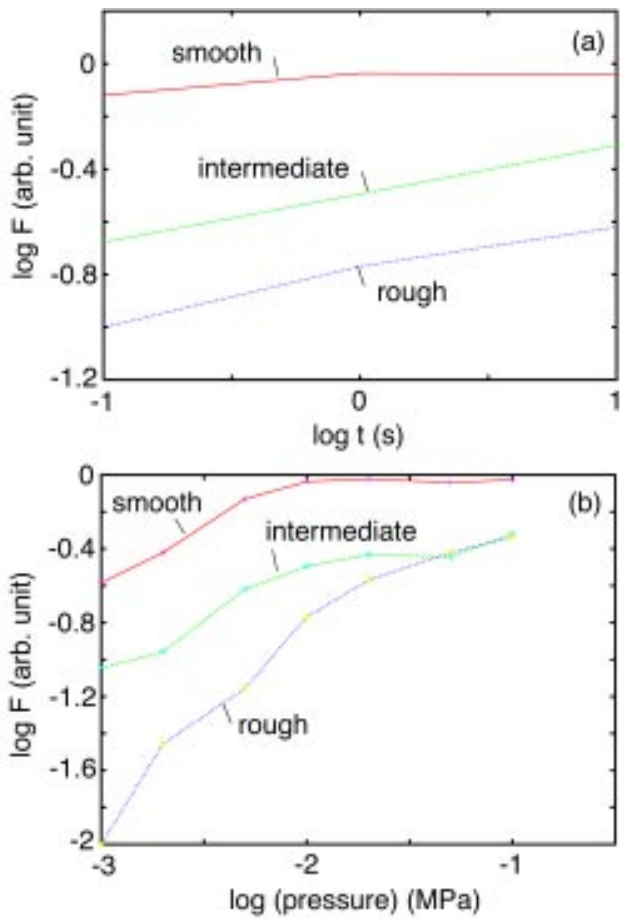

FIG. 8. (a) The logarithm of the pull-off force $F$ (in arbitrary units) as a function of the logarithm of the contact time. For the contact pressure $\sigma_{0}$ $=0.01 \mathrm{MPa}$. (b) The logarithm of the pull-off force [with the same units as in (a)] after $1 \mathrm{~s}$ of contact, as a function of the logarithm of the contact pressure. For three different surface roughnesses.

force $F$ on the contact time and contact pressure for smooth and rough PSA on the same smooth steel surface. The tack film is a standard polymer compound similar to PEHA. If one assumes, as a first approximation, that the pull-off force is proportional to the area of real contact, then if the smooth and rough PSA films in the measurements would correspond to the surfaces in Fig. 7, with the rms roughness 0.25 and 4 $\mu \mathrm{m}$, the agreement between the theory and the experiment is remarkably good (in Ref. 16 no numerical values of the rms roughness was presented for any of the studied surfaces). Nevertheless, one cannot expect that the pull-off force is strictly linearly related to the area of real contact. For example, at high pressure, complete contact occurs, but even in this case $F$ will depend on the contact time because of relaxation of the stress distribution at the interface (see below). Furthermore, many PSA are able to form chemical bonds with the surface over rather long times (around $24 \mathrm{~h}$ ). This situation is clearly excluded from our model.

It is clear from Fig. 7 and the experimental data reported on in Fig. 8 that in most cases (at room temperature) one would expect complete contact at the interface for pressures above $0.1 \mathrm{MPa}$, unless the surfaces are very rough or the contact time very short. However, even in situations where complete contact occur at the interface one may expect a dependence of the pull-off force on the contact time for the following reason: If the surfaces are squeezed together with a high pressure for a short time (leading to complete contact at the interface), a wide distribution of stresses may be expected at the interface. With increasing contact time (under a fixed squeezing pressure) the stress distribution will relax. 

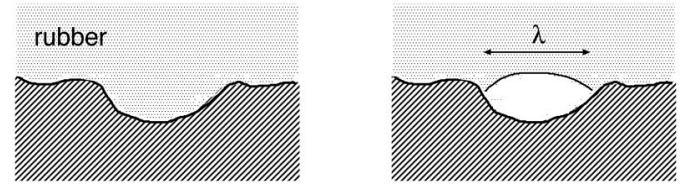

FIG. 9. A thermal fluctuation can give rise to a detached region at the interface if the local tensile stress is high enough.

When the applied contact pressure is removed, tensile stresses will develop in the rubber in the valleys of the rough substrate surface. If the contact time is long enough, these tensile stresses may be small and no local detachment will occur. However, if the contact time is short enough, very large tensile stresses will develop when the external squeezing stress is removed, which may lead to local detachment at the bottom of some valleys; see Fig. 9. These detached regions will then act as nucleation seeds for the cavities that usually are observed during the pull-off process. ${ }^{17,18}$ (In the discussion above we have implicitly assumed that the contact is perfect during the compression stage. However, it is possible that in some cases air pockets could be trapped in the valleys. These too could act as seeds for cavities during pull-off. ${ }^{19}$ )

Let us study the stress relaxation at the interface when the solids are squeezed into complete contact at $t=0$. For a purely elastic solid the stress distribution $P(\sigma, \zeta)$ at the magnification $\zeta$ is (approximately) given by

$$
P(\sigma, \zeta) \approx \frac{P_{1}}{2 \sigma_{0}} \exp \left[-\left(\frac{\sigma}{\sigma_{0}}-1\right)^{2} \frac{\pi}{4} P_{1}^{2}\right],
$$

where $P_{1}=P_{1}(\zeta)$ is given by (4a). Following the earlier treatment, we will assume that this formula is approximately valid also for a viscoelastic solid if we replace $P_{1}(\zeta)$ with $P_{1}(\zeta, t)$ given by $(41)$. Thus, the stress distribution is approximately Gaussian with the root-mean-square width,

$$
\sigma_{1}(\zeta, t)=\left[\left\langle\left(\sigma-\sigma_{0}\right)^{2}\right\rangle\right]^{1 / 2}=\left(\frac{2}{\pi}\right)^{1 / 2} \frac{\sigma_{0}}{P_{1}(\zeta, t)} .
$$

Note that $P_{1} \sim \sigma_{0}$ so that $\sigma_{1}$ is independent of the squeezing pressure $\sigma_{0}$, which must be the case when complete contact occurs at the interface. In Fig. 10 we show $\sigma_{1}$ (the root mean square of the fluctuating stress at the interface) for PEHA$\mathrm{AA}$, at the magnification $\zeta=10^{5}$ corresponding to the wavelength $\lambda \approx 1 \mathrm{~nm}$, as a function of the logarithm of the contact time. The results are for steel surfaces with the rms roughness amplitudes $h_{0}=1,5$, and $10 \mu \mathrm{m}$, and for the temperature $T=20^{\circ} \mathrm{C}$. The calculations have been performed for the squeezing pressure $\sigma_{0}=1 \mathrm{MPa}$, which is high enough to give complete contact at the interface for the roughness amplitudes and contact times displayed in Fig. 10. When the applied stress is reduced to zero, the probability $\Phi(\sigma)$ to find a stress smaller than $\sigma$ can be obtained from (42) with $\sigma_{0}$ $=0$ :

$$
\Phi(\sigma)=\frac{1}{(2 \pi)^{1 / 2}} \int_{-\infty}^{\sigma / \sigma_{1}} d x e^{-x^{2} / 2} .
$$

Since there are roughly $N=A_{0} / \lambda^{2}$ valleys on the substrate surface area $A_{0}$, the number of valleys with tensile stresses

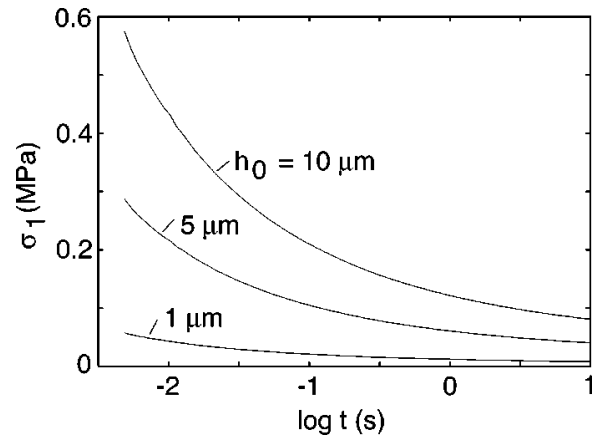

FIG. 10. The root-mean-square (rms) $\sigma_{1}$ of the fluctuating stress at the interface for PEHA-AA, at the magnification $\zeta=10^{5}$ corresponding to the wavelength $\lambda \approx 1 \mathrm{~nm}$, as a function of the logarithm of the contact time. For a self-affine fractal substrate surface with the fractal dimension $D_{f}=2.2$, the roll-off wave vector $q_{0}=6 \times 10^{4} \mathrm{~m}^{-1}$ and rms roughness amplitudes $h_{0}$ $=1,5$, and $10 \mu \mathrm{m}$, and for the temperature $T=20^{\circ} \mathrm{C}$ and contact pressure $\sigma_{0}=1 \mathrm{MPa}$.

higher than $-\sigma($ note $\sigma<0)$ will be $N \Phi(\sigma)$. For example, if $|\sigma| / \sigma_{1}=5$ and $A_{0}=1 \mathrm{~cm}^{2}$ and $\lambda=1 \mathrm{~nm}$, we get $\Phi \approx 10^{-7}$ and $N \Phi \approx 10^{7}$.

Assume that the local tensile stress $\sigma$ act in a small region of order $\lambda \times \lambda$ at the interface. Let us calculate the change in the free energy when this region undergoes detachment; see Fig. 9. Using standard crack theory we get

$$
U \approx \Delta \gamma \lambda^{2}-\sigma^{2} \lambda^{3} / E_{0},
$$

where $E_{0}=E(\omega)$ for $\omega=0$, is the low-frequency elastic modulus. This quantity is negative, i.e., the detached state has a lower free energy than the attached state, if

$$
|\sigma|>\sigma^{*}=\left(\frac{E_{0} \Delta \gamma}{\lambda}\right)^{1 / 2} .
$$

The barrier separating the attached state from the detached state has the height

$$
\Delta U=5 \Delta \gamma \lambda^{2} / 9 \text {. }
$$

If $\lambda=1 \mathrm{~nm}, \Delta \gamma \approx 3-5 \mathrm{meV} / \AA^{2}$ and $E=10^{4} \mathrm{~Pa}$, we get $\sigma^{*}$ $=0.6 \mathrm{MPa}$. If the local tensile stress takes at least this value, the detached state will have smaller energy than the attached state. However, only if the energy barrier that separates the complete contact state from the (locally) detached state is smaller than, say, $\sim 1 \mathrm{eV}$, will the system be able to jump (by a thermal fluctuation) over the barrier on typical macroscopic times, say $1 \mathrm{~min}$. Using (43) we calculate the barrier height $\Delta U \approx 0.2 \mathrm{eV}$ when $\lambda \approx 1 \mathrm{~nm}$, while $\Delta U \sim 1 \mathrm{eV}$ when $\lambda$ is of the order of a few nanometers. Thus, nanometer-sized detached regions (which may act as a nucleus for the cavities formed during pull-off) can form spontaneously if the tensile stress at the interface (in the absence of a squeezing pressure) becomes of order $0.6 \mathrm{MPa}$, which will be the case if the surface roughness is large enough and the contact time short enough. For example, if the rms roughness amplitude equal $h_{0}=10 \mu \mathrm{m}$, then for the PEHA-AA adhesive with the contact time $1 \mathrm{~s}$ we get (see Fig. 10) $\sigma_{1} \approx 0.12 \mathrm{MPa}$. Thus, with $|\sigma|=0.6 \mathrm{MPa}$ we get $|\sigma| / \sigma_{1}=5$ and $\Phi \approx 10^{-7}$. If the contact area is $1 \mathrm{~cm}^{2}$, as in the example above, we get $N \Phi \approx 10^{7}$ sites 


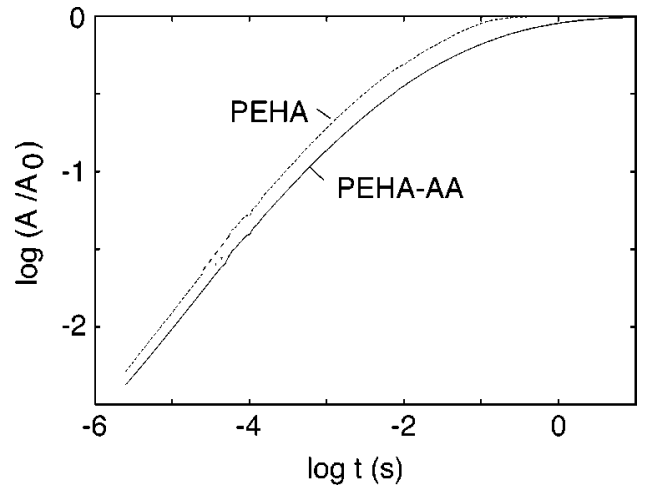

FIG. 11. The logarithm of the relative contact area for PEHA (dashed line) and PEHA-AA (solid line), at the magnification $\zeta=10^{5}$ corresponding to the wavelength $\lambda \approx 1 \mathrm{~nm}$, as a function of the logarithm of the contact time. For a self-affine fractal substrate surface with the fractal dimension $D_{f}=2.2$, the roll-off wave vector $q_{0}=6 \times 10^{4} \mathrm{~m}^{-1}$ and rms roughness amplitudes $h_{0}$ $=20 \mu \mathrm{m}$, and for the temperature $T=20^{\circ} \mathrm{C}$ and contact pressure $\sigma_{0}$ $=0.4 \mathrm{MPa}$.

at the interface where (nanometer-sized) detached regions can be formed already without any applied pull-off stress.

It is important to note that when complete contact occur at the interface, the nucleation of a detached region can only occur by a thermal fluctuation. Thus, the local tensile stress necessary for detachment (in the absence of thermal fluctuations) is of order $\Delta \gamma / a \approx 100 \mathrm{MPa}$ (where $a$ is an atomic distance), which is much higher than the tensile stress during pull-off, which usually is below $1 \mathrm{MPa}$. Thus, the complete contact state can only be broken by (local) thermal fluctuations.

As shown above, for pressures of order $\sim 0.1 \mathrm{MPa}$ or higher, and contact times of order $1 \mathrm{~s}$ or more, complete contact will occur at the interface unless the surface roughness amplitude of the substrate is very high. Most experiments performed on tack are for substrate surface roughness below a few $\mu \mathrm{m}$, but many surfaces of practical importance have much larger roughness amplitudes. Here we present some numerical results for very rough surfaces and for high squeezing pressures.

Figure 11 shows the logarithm of the relative contact area for PEHA (dashed line) and for PEHA-AA (solid line), as a function of the logarithm of the contact time. The results are for a "steel" substrate surface with the rms roughness $h_{0}=20 \mu \mathrm{m}$, at the highest magnification $\zeta=10^{5}$, corresponding to the wavelength $\lambda \approx 1 \mathrm{~nm}$. The temperature $T$ $=20^{\circ} \mathrm{C}$, and the squeezing pressure $\sigma_{0}=0.4 \mathrm{MPa}$. In this case complete contact is reached after about $0.1 \mathrm{~s}$ of contact for PEHA and $1 \mathrm{~s}$ of contact for PEHA-AA.

Figure 12 shows the relative contact area as a function of the logarithm of the magnification. Results are again shown for PEHA (dashed lines) and PEHA-AA (solid lines) for $T$ $=20^{\circ} \mathrm{C}$, and for two different "steel" substrates with the rms roughness amplitudes, $h_{0}=20$ and $100 \mu \mathrm{m}$. In a practical application the tack film may be of order $100 \mu \mathrm{m}$ thick, in which case the present result obtained for a semi-infinite viscoelastic solid may be of only qualitative validity.

Figure 13 shows the relative contact area for PEHA-AA as a function of the logarithm of the magnification for the

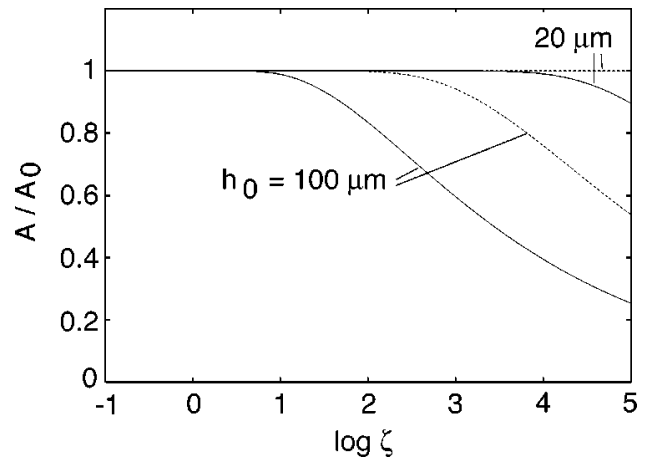

FIG. 12. The relative contact area as a function of the logarithm of the magnification for a self-affine fractal substrate surface with the fractal dimension $D_{f}=2.2$ and the roll-off wave vector $q_{0}=6 \times 10^{4} \mathrm{~m}^{-1}$. Results are shown for PEHA (dashed lines) and PEHA-AA (solid lines) for $T=20^{\circ} \mathrm{C}$, for two different substrate rms roughness amplitudes, $h_{0}=20 \mu \mathrm{m}$ and 100 $\mu \mathrm{m}$. After $1 \mathrm{~s}$ of contact at the pressure $\sigma_{0}=0.4 \mathrm{MPa}$.

temperatures $T=-20^{\circ} \mathrm{C}, 0{ }^{\circ} \mathrm{C}$, and $20^{\circ} \mathrm{C}$. As expected, the contact area decreases with decreasing temperature; at very low temperature (not shown) the polymer film is in a hard glassy state and the contact area is very small.

\section{B. Rubber friction}

Many sliding systems exhibit a stiction spike, i.e., a start-up friction force that is higher than the steady-state friction force, as illustrated in Fig. 14. The height $\Delta F$ of the stiction spike depends on many parameters, e.g., the time period the solids have been in contact with before sliding, the sliding or pull-velocity, and the temperature. ${ }^{20}$ Very many different physical processes can give rise to a stiction spike. For example, for two polymers in contact, chain interdiffusion may lead to a stiction spike. Another mechanism that has been observed for very many materials, e.g., metals, paper, stone, glass and glassy polymers, is a slow increase in the contact area between the solids as a result of thermally induced plastic flow (creep), which always will occur when the local stress in the contact areas is close to the plastic

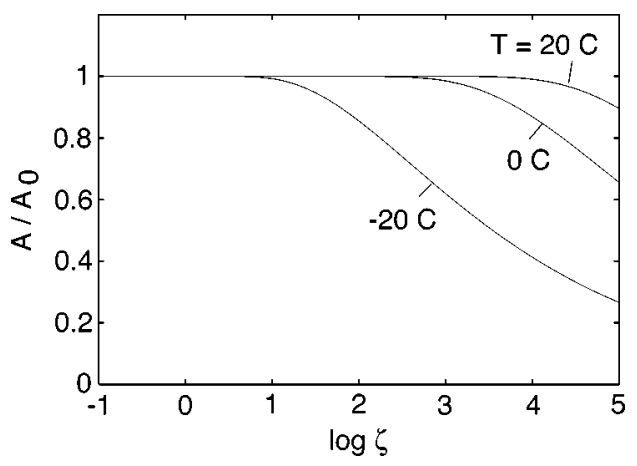

FIG. 13. The relative contact area for PEHA-AA as a function of the logarithm of the magnification for a self-affine fractal substrate surface with the fractal dimension $D_{f}=2.2$, the roll-off wave vector $q_{0}=6 \times 10^{4} \mathrm{~m}^{-1}$, and the rms roughness amplitudes $h_{0}=20 \mu \mathrm{m}$. Results are shown for the temperatures $T=-20^{\circ} \mathrm{C}, 0{ }^{\circ} \mathrm{C}$, and $20^{\circ} \mathrm{C}$. After $1 \mathrm{~s}$ of contact at the pressure $\sigma_{0}=0.4 \mathrm{MPa}$. 


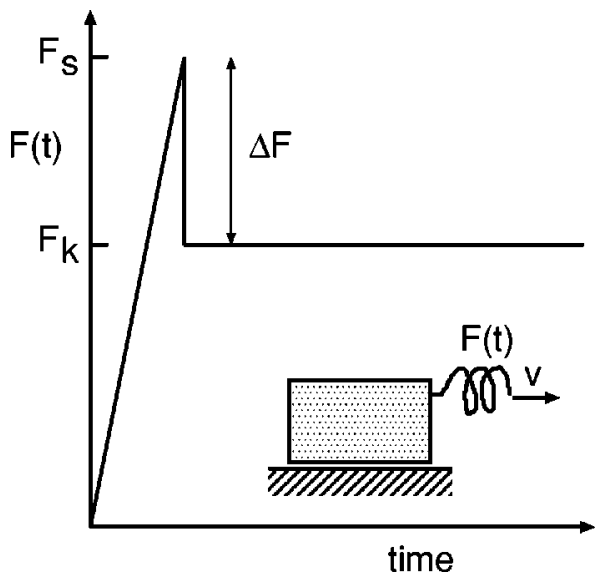

FIG. 14. A block is pulled on a substrate. The spring force is shown as a function of time. The height $\Delta F$ of the stiction spike depends on the time $t$ of stationary contact.

yield stress of the solids. Experiment ${ }^{21}$ and theory ${ }^{20,22}$ have shown that the area of real contact increases with the contact time $t$ according to

$$
A(t) \approx a+b \ln (1+t / \tau),
$$

where $\tau$ is a characteristic time. If one assume that the shear stress $S$ necessary to break the contact junctions depends linearly on the perpendicular pressure, $S=\alpha+\beta P$, it follows that the stiction spike $\Delta F$ increases roughly logarithmically with the contact time, as is indeed observed in most cases.

The contact area between a rubber block and a hard, rough, substrate also increases roughly logarithmically with the contact time (as long as the contact area is small compared to the nominal contact area). This is illustrated in Figs. 15 for tire tread rubber in contact with an asphalt road. (Figure 16 shows for the same system the dependence of the contact area on the magnification.) However, for rubber the physical origin of this enhancement of the contact area is very different from that for most other solids since rubber does usually not yield plastically, but rather deforms purely elastically. For rubber, the time dependence of the contact

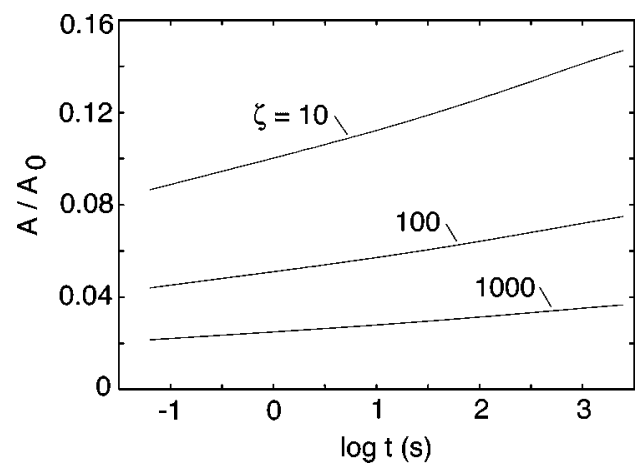

FIG. 15. The relative contact area as a function of the logarithm of the contact time for a tread tire rubber at the temperature $T=60^{\circ} \mathrm{C}$, and for the nominal pressures $\sigma_{0}=0.4 \mathrm{MPa}$. Results are shown for three different magnifications: $\zeta=10,100$, and 1000 . The surface power spectra correspond to an asphalt track with $0.26 \mathrm{~mm}$ root-mean-square roughness, and the roll-off wave vector $q_{0}=1694 \mathrm{~m}^{-1}$, so that the magnification $\zeta=1$ corresponds to the wavelength $\lambda_{0}=2 \pi / q_{0} \approx 0.4 \mathrm{~cm}$. Note that the contact area increases with about $50 \%$ when the contact time increases from $1 \mathrm{~s}$ to $1 \mathrm{~h}$.

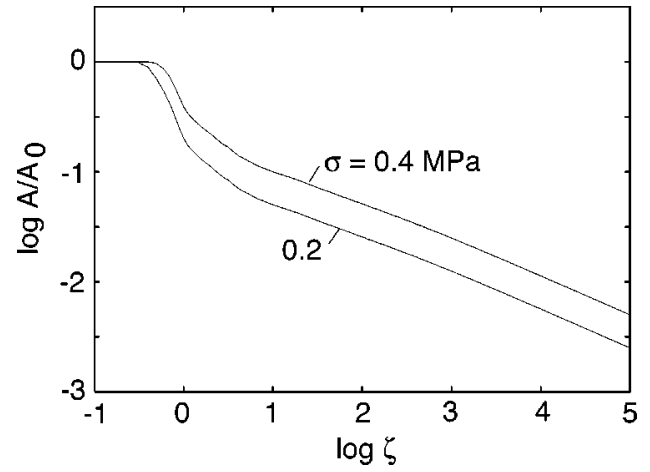

FIG. 16. The relative contact area after one second of contact, as a function of the magnification, where $\zeta=1$ corresponds to the wavelength $\lambda_{0}$ $=2 \pi / q_{0} \approx 0.4 \mathrm{~cm}$. The highest magnification $\zeta=10^{5}$ corresponds to $\lambda$ $\approx 0.04 \mu \mathrm{m}$. Results are shown for the nominal pressures $\sigma_{0}=0.2$ and 0.4 $\mathrm{MPa}$. For the same tread tire rubber and surface roughness power spectra as in Fig. 15, at the temperature $T=60^{\circ} \mathrm{C}$.

area is due to thermally activated flips of polymer segments. That is, the segments of the polymer chains can take many different positions or orientations, which correspond to different local minima of the total potential $V$ of the polymer system. At a given temperature the polymer segments perform thermally activated jumps between these local minima. When an external force act on a rubber block, it will deform the potential energy surface in such a way that the local potential minima become deeper for such chain configurations that correspond to an elongation of the rubber block in the direction of the external force. The polymer segments will spend more time in the deeper minima, which correspond to a macroscopic deformation of the rubber along the direction of the external force. A glassy (disordered) material such as rubber has a very wide distribution of barrier heights, separating the local minima. Since a very long waiting time is necessary in order for a thermal fluctuation to be able to flip a polymer segment over a high potential energy barrier, the wide distribution of barrier heights gives rise to the wide distribution of relaxation times observed for most rubberlike materials, and to the asymptotically very slow (approximately logarithmic) increase in the contact area with the contact time.

Does rubber friction exhibit stiction spikes due to the (slow) increase in the contact area? At first one may think that the increase in the contact area with the time of stationary contact observed for rubber will give rise to stiction peaks, just as is observed for metals and other materials that yield plastically in the contact areas (see above). However, the situation for rubber is more complex because the origin of the increase in the contact area is different for rubber than for most other solids.

In an earlier publication one of us has developed a theory of rubber friction for nonstationary sliding on a hard (randomly rough) substrate, ${ }^{23,24}$ but neglecting the flash temperature (see below). For this case no stiction spike was observed when the sliding velocity was abruptly increases from a value $v_{1}$ to a higher value $v_{2}$, as long as both $v_{1}$ and $v_{2}$ are below the velocity $v_{c}$ for which the steady-state kinetic friction coefficient $\mu_{k}(v)$ is maximal. In this theory rubber fric- 

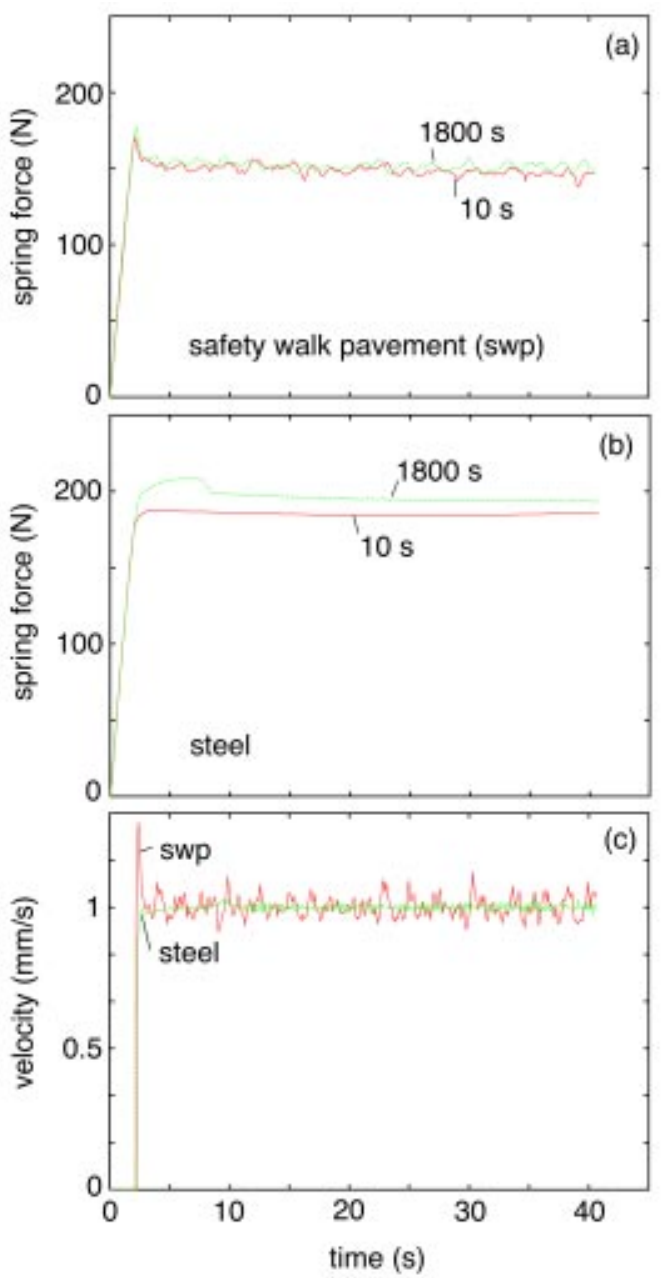

FIG. 17. A rubber block (in the form of a cylinder with the diameter $29 \mathrm{~mm}$ ) with the vertical load $F_{N}=132 \mathrm{~N}$ is pulled on (a) a safety walk pavement, and (b) a steel surface. The spring force (i.e., the force in the pulling wire) is shown as a function of time, starting from the equilibrium state prepared by having the surfaces in stationary contact for 10 and $1800 \mathrm{~s}$; we refer to these time periods as the waiting time. In (c) we show the rubber block velocity as a function of time (for the $1800 \mathrm{~s}$ waiting time). The experiments were performed at room temperature using a standard tire tread rubber.

tion is attributed to the bulk viscoelasticity of the rubber, and $\mu_{k} \rightarrow 0$ as $v \rightarrow 0$ as a consequence of the fact that $\operatorname{Im} E(\omega)$ $\rightarrow 0$ as $\omega \rightarrow 0 .{ }^{25}$ Thus, we can imagine a limiting case where both the force acting on the rubber block, and the sliding velocity, are arbitrary small before it is abruptly increased to a value $v_{2}$ [below the maximum of the $\mu_{k}(v)$ curve]. ${ }^{26}$ For this case the rubber friction theory ${ }^{23}$ would predict no stiction spike. The physical reason for this is that the area of contact gradually decreases during the (tangential) load phase, and when full sliding occurs the contact area is already reduced to the value it has during steady sliding at the given sliding velocity. Since this situation is very similar to the situation described above, involving a rubber block in stationary contact with the substrate for some given waiting time before sliding, it is plausible to assume that no stiction spike may occur in a stop-start experiment at very low pulling velocity. This result is confirmed by the experiments reported on in Ref. 27 and below. Thus, in Fig. 17 we show the sliding dynamics when a rubber block (in the form of a cyl- inder with the diameter $29 \mathrm{~mm}$ ) with the vertical load $F_{N}$ $=132 \mathrm{~N}$ is pulled on (a) a safety walk pavement, and (b) a steel surface. The spring force (i.e., the force in the pulling wire) is shown as a function of time, starting from the equilibrium state prepared by having the surfaces in stationary contact for 10 and $1800 \mathrm{~s}$; we refer to these time periods as the waiting time. In (c) we show the rubber block velocity as a function of time (for the case of $1800 \mathrm{~s}$ waiting time).

Note that for the safety walk paper (a) the spring force is nearly independent of the waiting time. For the smooth steel surface no stiction spike is observed, but the kinetic friction coefficient for $t>10 \mathrm{~s}$ is about $8 \%$ higher after $1800 \mathrm{~s}$ contact time, as compared to the case of $10 \mathrm{~s}$ contact time. We attribute this to the following effect: before sliding the surface of the rubber block was abraded with sandpaper to remove the top rubber surface layer, which may have different properties from the underlying rubber, but which is of no interest in tire application as it is removed after a short run-in time period. As a result the rubber surface may be rougher than the polished steel surface. When the rubber is in stationary contact with the steel surface, the rubber-steel asperity contact areas will increase with time because of the viscoelastic flow of the rubber (see above). If the steel surface is much smoother than the rubber surface, then during sliding the rubber will mainly make contact with the steel in the same rubber surface regions as during the stationary time period. Thus, even during sliding the rubber-steel contact area will be largest for the case with the longest waiting time period. This will increase the rubber friction, in particular, if there is a contribution from the adhesive rubber-steel interaction (which depends on the area of real contact). The increased friction during the first $5 \mathrm{~mm}$ of sliding distance in (b) (1800 s contact time) cannot be a flash temperature effect, since then it should also be observed for the $10 \mathrm{~s}$ waiting time case. Finally, we note that the present data shows that there is no contribution to the stiction spike from pinning effects at the rubber-substrate interface arising from thermally activated rearrangement of the rubber polymer chains in the substrate potential during the time of stationary contact. This is an important and nontrivial result. ${ }^{28}$

\section{Seals}

Surface roughness is an important factor that influences the rate of leakage through seals. Vacuum seals are a special case where the limit on the leakage rate is particularly rigorous. The exact mechanism of the roughness-induced leakage is not well understood. ${ }^{29}$ In this section we present a new way of looking at this problem.

Viscoelastic materials such as rubber are often used for sealing. Here we consider the tire-rim sealing. The rim is made from steel. We assume that the steel surface is a selfaffine fractal for $q>q_{0}$, with the fractal exponent $D=2.2$ (i.e., $H=0.8$ ). The surface root-mean-square roughness is assumed to be $1 \mu \mathrm{m}$, as is typical for polished steel surfaces. The long distance roll-off wave vector is assumed to be $q_{0}$ $=6 \times 10^{4} \mathrm{~m}^{-1}$, corresponding to the wavelength $\lambda_{0}$ $=2 \pi / q_{0} \approx 100 \mu \mathrm{m}$. The power spectra for the surface is shown in Fig. 5. 

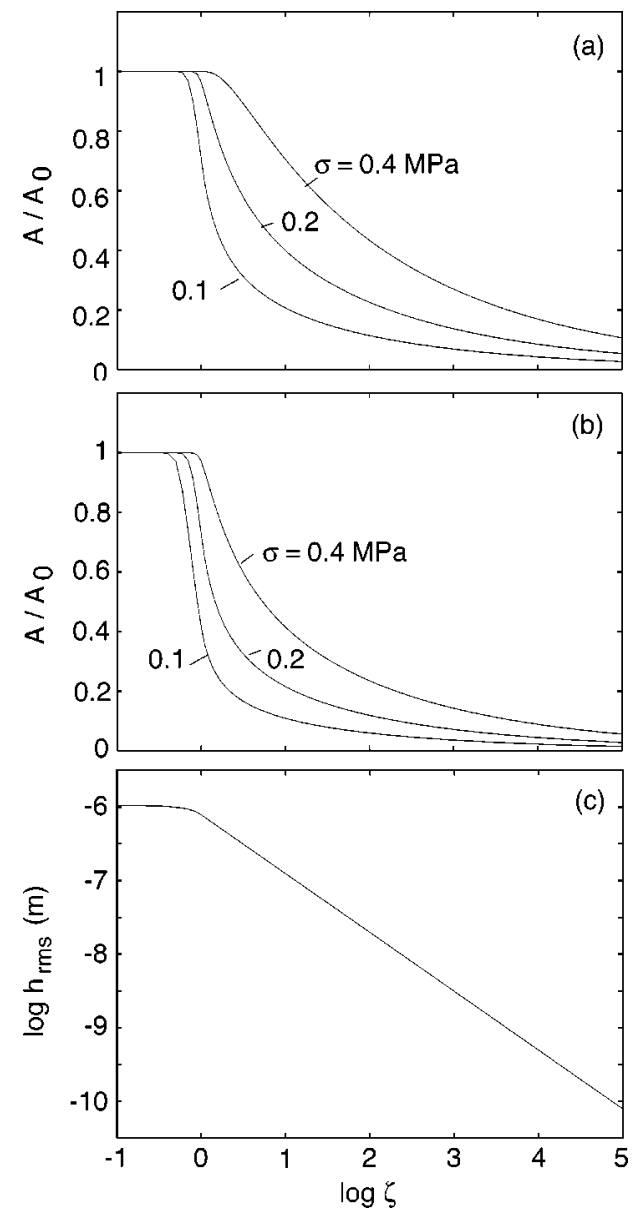

FIG. 18. The relative contact area after one second of contact (a) (for tread tire rubber), (b) (for rim tire rubber), and the root-mean-square roughness (c), as a function of the magnification, where $\zeta=1$ corresponds to the wavelength $\lambda_{0}=2 \pi / q_{0} \approx 100 \mu \mathrm{m}$. The highest magnification $\zeta=10^{5}$ correspond to $\lambda \approx 1 \mathrm{~nm}$. Results are shown for the nominal pressures $\sigma_{0}=0.1,0.2$, and $0.4 \mathrm{MPa}$, at the temperature $T=60^{\circ} \mathrm{C}$. For substrate surface with the rms roughness, $h_{0}=1 \mu \mathrm{m}$ and the roll-off wave vector $q_{0}=6 \times 10^{4} \mathrm{~m}^{-1}$.

Figure 18 shows the relative contact area after one second of contact for tread tire rubber (a), and for rim tire rubber (b), and the root-mean-square roughness (c), as a function of the magnification. Here we have defined

$$
h_{\mathrm{rms}}=\left(2 \pi \int_{\zeta q_{0}}^{q_{1}} d q q C(q)\right)^{1 / 2} .
$$

The results are for the nominal pressures $\sigma_{0}=0.1,0.2$, and $0.4 \mathrm{MPa}$, at the temperature $T=60^{\circ} \mathrm{C}$. Figure 19 shows the relative contact area (at the highest magnification $\zeta=10^{5}$ ) as a function of the logarithm of the contact time $t$, for the nominal pressures $\sigma_{0}=0.1 \mathrm{MPa}$.

We now study the rubber-steel interface at an increasing magnification. At the lowest magnification $\zeta<1$ complete contact occurs at the interface; see Fig. 20 (left). When we increase the magnification, we observe noncontact areas or islands. The magnification is now increased until the noncontact area percolates, i.e., until a channel of noncontact surface area, extending from the high-pressure internal region of the tire to the outside (atmospheric pressure region), is first observed. As the magnification is increased further, new noncontact region will be observed, but the separation between

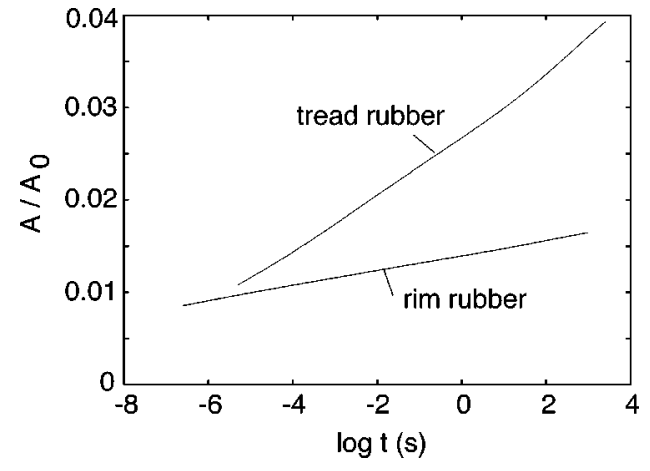

FIG. 19. The relative contact area at the highest magnification, $\zeta=10^{5}$ (corresponds to $\lambda \approx 1 \mathrm{~nm}$ ) as a function of the logarithm of the contact time $t$. For the nominal pressures, $\sigma_{0}=0.1 \mathrm{MPa}$. For a tread tire rubber compound, and for a compound used in the tire-rim area, at the temperature $T$ $=60^{\circ} \mathrm{C}$. The substrate is self-affine fractal with the fractal dimension $D$ $=2.2$, and with the rms roughness $1 \mu \mathrm{m}$, and with $q_{0}=6 \times 10^{4} \mathrm{~m}^{-1}$.

the surfaces in these new noncontact areas will be smaller than along the percolation channel. Since the gas flow $\dot{N}$ (number of molecules per unit time) through a rectangular pore of height $h$ depends as $\dot{N} \sim h^{3}$ (see below) we will assume that most gas will leak through the percolation channel.

Assuming that the contact regions at any magnification are approximately randomly distributed in the apparent contact region, we expect from percolation theory that the noncontact region will percolate when $A / A_{0} \approx 1-p_{c}$, where $p_{c}$ is the site percolation number. ${ }^{30}$ For a hexagonal lattice, which is the most plausible lattice structure in the present case, one has $p_{c} \approx 0.7$, while for a square lattice (as in Fig. 20) percolation occurs at $p_{c} \approx 0.6$. Thus, the exact value of the percolation threshold does not depend sensitively on the symmetry of the unit cell.

We assume that the main gas leakage comes from gas flow through the percolation channel. The most narrow pass in this channel can be considered as a rectangular pore of height $h$, and of width and length $\lambda$, where $\lambda$ is determined by the magnification $\zeta_{c}$ at the point where $A / A_{0} \approx 0.3$. The height $h$ of the pore is determined by the rms roughness at the magnification $\zeta_{c}$. In the present case, if the tire gas pressure is in the range $0.2-0.3 \mathrm{MPa}$, from Fig. 18(b) we get $\zeta_{c} \approx 10$ and from Fig. 18(c), $h \approx 0.1 \mu \mathrm{m}$.

We divide the tire-rim contact area into $m$ square areas $B \times B$, where $B$ is the width of the tire-rim contact area (we
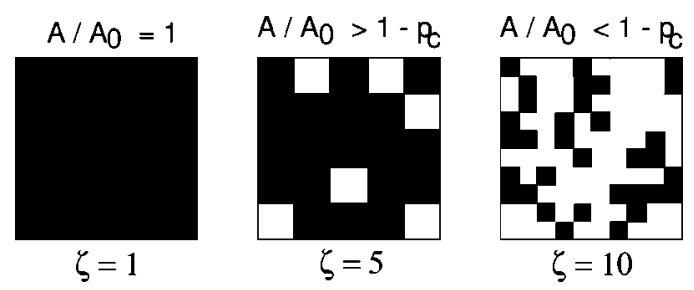

FIG. 20. When the interface between the solids is studied at low magnification, there appears to be complete contact (black area) between the solids. When the magnification is increased it is observed that only partial contact occur. At high enough magnification the noncontact (white) surface area will percolate and one (or several) airflow channels will be visible at the interface. 
expect $B$ to be of order a few $\mathrm{cm}$ ). The number of squares is $m=2 \pi R / B$, where $R$ is the radius of the tire at the rim. We expect $m \approx 100$.

Let us study the gas flow through a rectangular pore of height $h$ and width and length (in the flow direction) $\lambda$. We assume stationary and laminar flow, and that $h \ll \lambda$. In this case the basic equations of hydrodynamics become

$$
\begin{aligned}
& \boldsymbol{\nabla} P \approx \mu \nabla^{2} \mathbf{v}, \\
& \boldsymbol{\nabla} \cdot(n \mathbf{v}) \approx 0,
\end{aligned}
$$

where $P$ is the gas pressure, $\mathbf{v}$ the flow velocity, $\mu$ the viscosity, and $n$ the gas number density. We assume constant temperature, and that the pressure $P$ is related to the number density $n$ via the ideal gas law:

$$
P=n k_{\mathrm{B}} T \text {. }
$$

Using the equations above the number of molecules that flow through the pore per unit time is

$$
\dot{N} \approx \frac{\left(P_{1}^{2}-P_{0}^{2}\right) h^{3}}{24 \mu k_{\mathrm{B}} T},
$$

where $P_{1}$ and $P_{0}$ are the pressure inside and outside the tire, respectively. Here we have implicitly assumed that the full pressure drop $P_{1}-P_{0}$ occur over the pore. If $N_{1}(t)$ denote the number of gas phase molecules in the tire (which is proportional to the pressure $P_{1}$ in the tire), we get for the typical case $P_{1} \gg P_{0}$ :

$$
\dot{N}_{1} \approx-\frac{m P_{1}^{2} h^{3}}{24 \mu k_{\mathrm{B}} T} .
$$

Thus, the time it takes for the pressure in the tire to drop with $\approx 4 \%$ is

$$
\Delta t=\frac{\mu}{P_{1}} \frac{V_{1}}{h^{3}},
$$

where $V_{1}=V_{0} / m$ (where $V_{0}$ is the air volume in the tire) is the volume of air in an angular section of the tire of width $B$. With $B \approx 3 \mathrm{~cm}$ we get $V_{1} \approx 3 \times 10^{-4} \mathrm{~m}^{3}$, and using the viscosity of air $\mu \approx 17 \times 10^{-6} \mathrm{Ns} / \mathrm{m}^{2}$ gives $\Delta t \approx 1$ year. This is an upper limit of the leakage time, since when the interfacial contact area is studied at higher magnification new pore channels through which the air can leak will be detected. The discussion above is only valid if the mean-free path for the gas phase molecules (at the pressure $P_{1}$ ) is shorter than the pore height $h$. If this is not the case the hydrodynamic description presented above is not valid, and one must take into account the nonspecular scattering of the gas phase molecules from the pore surfaces.

It is interesting to note that the adhesional interaction between the rubber surface and the steel rim is likely to have negligible influence on the leakage rate. Adhesion will affect the (apparent) contact area only at very high magnification (see Sec. II), but most of the gas leakage occur via the much larger air flow channels, which can be observed at low magnification.

\section{SUMMARY AND CONCLUSION}

In this paper we have studied the time-dependent contact area as a viscoelastic solid is squeezed against a randomly rough substrate. Using a recently developed contact mechanics theory we presented results for the dependence of the contact area on time and on the magnification $\zeta$. All numerical results were obtained for self-affine fractal surfaces. Detailed applications to tack, rubber friction, and sealing have been presented, and compared with experimental data.

The theory we describe can only be applied when the contact area increases with time, which always will be the case if the system is exposed to a constant squeezing pressure for time $t>0$. The detailed behavior of the tack film during pull-off is a much more complicated topic in general involving bond breaking, cavity formation, stringing, and highly nonlinear elongation processes. This topic cannot be studied analytically with the same accuracy as the contact formation process discussed in this paper. Nevertheless, detailed information about the contact area as a function of magnification and contact time, and information about the stress distribution at the interface at the onset of pull-off, forms a necessary background for the discussion of the tackfilm dynamics during pull-off.

The contact mechanics theory presented above neglect the adhesional interaction between the solids. ${ }^{31}$ We believe that this is a good approximation in most of the applications presented in this paper involving relative rough substrates. However, following the theory recently developed for elastic solids, ${ }^{8}$ it should be possible to generalize the viscoelastic contact mechanics theory to include the adhesional interaction.

\section{ACKNOWLEDGMENTS}

The rubber friction and contact mechanics results presented in this paper is the result of an ongoing collaboration between B.P. and Pirelli. B.P. thanks Pirelli for support, and for many useful discussions with the Pirelli tire research and development team. He also thanks G. Heinrich and M. Klüppel for interesting discussions.

\section{APPENDIX: SIMPLE DERIVATION OF THE JKR EQUATIONS}

Here we illustrate how the concepts of the adhesion pressure can be used to obtain the standard $\mathrm{JKR}^{32}$ result for an elastic sphere (radius $R$ ) adhering to a flat rigid surface. In the absence of an applied load, the radius of the contact area, according to the JKR theory, is

$$
r=\left(\frac{9 \pi R^{2} \gamma}{2 E^{*}}\right)^{1 / 3} .
$$

This equation can be derived using the adhesion pressure arguments as follows: When an elastic ball is squeezed against a flat without adhesion, the Hertz theory gives a circular contact area with the radius

$$
r=\frac{3 \pi R \sigma_{0}}{4 E^{*}},
$$


where $\sigma_{0}=F /\left(\pi r^{2}\right)$ is the nominal (or average) squeezing pressure. To obtain the contact radius for the case of adhesive contact without a squeezing force we must, according to the discussion in Sec. II, just replace the squeezing pressure $\sigma_{0}$ with the adhesion pressure $\sigma_{a}$ given by

$$
\sigma_{a}=\left(\frac{\pi \gamma E^{*}}{\lambda}\right)^{1 / 2},
$$

where the lateral resolution $\lambda$ now corresponds to the diameter $2 r$ of the contact area (we need to go to this resolution before we can observe that there actually is contact between the two objects). Substituting (A3) in (A2) gives

$$
r=\left(\frac{9 \pi^{3} R^{2} \gamma}{32 E^{*}}\right)^{1 / 3}
$$

which, except for a factor $\left(\pi^{2} / 16\right)^{1 / 3} \approx 0.9$, agree with $(\mathrm{A} 1)$. The pull-off force is given by the product between the adhesion stress and the contact area:

$$
F_{\text {pull-off }} \approx \sigma_{a} \pi r^{2}=\frac{3 \pi^{3}}{16} \gamma R,
$$

which is the JKR result to within a factor $\pi^{2} / 8 \approx 1.2$.

${ }^{1}$ J. A. Greenwood, in Fundamentals of Friction, Macroscopic and Microscopic Processes, edited by I. L. Singer and H. M. Pollack (Kluwer, Dordrecht, 1992); J. A. Greenwood and J. B. P. Williamson, Proc. R. Soc. London, Ser. A 295, 300 (1966)

${ }^{2}$ C. Creton and L. Leibler, J. Polym. Sci., Part B: Polym. Phys. 34, 545 (1996).

${ }^{3}$ C. Y. Hui, Y. Y. Lin, and J. M. Baney, J. Polym. Sci., Part B: Polym. Phys. 38, 1485 (2000)

${ }^{4}$ J. F. Archard, Proc. R. Soc. London, Ser. A 243, 190 (1957); K. L. Johnson, Contact Mechanics (Cambridge University Press, Cambridge, 1985).

${ }^{5}$ B. N. J. Persson, F. Bucher, and B. Chiaia, Phys. Rev. B 65, 184106 (2002).

${ }^{6}$ B. N. J. Persson, J. Chem. Phys. 115, 3840 (2001).

${ }^{7}$ B. N. J. Persson, Phys. Rev. Lett. 87, 116101 (2001).

${ }^{8}$ B. N. J. Persson, Eur. Phys. J. E 8, 385 (2002).

${ }^{9}$ H. Lakrout, P. Sergot, and C. Creton, J. Adhes. 69, 307 (1999).

${ }^{10}$ C. Creton, J. C. Hooker, and K. R. Shull, Langmuir 17, 4948 (2001).

${ }^{11}$ K. Brown, J. C. Hooker, and C. Creton, Macromol. Mat. Eng. 287, 163 (2002).
${ }^{12}$ K. L. Johnson, Contact Mechanics (Cambridge University Press, Cambridge, 1985).

${ }^{13}$ A. W. Bush, R. D. Gibson, and T. R. Thomas, Wear 35, 87 (1975); A. W. Bush, R. D. Gibson, and G. P. Keogh, Mech. Res. Commun. 3, 169 (1976).

${ }^{14}$ J. Feder, Fractals (Plenum, New York, 1988); M. V. Berry and Z. V. Lewis, Proc. R. Soc. London, Ser. A 370, 459 (1980).

${ }^{15}$ B. N. J. Persson (unpublished).

${ }^{16}$ F. H. Hammond, ASTM Spec. Tech. Publ. 360, 123 (1964).

${ }^{17}$ K. R. Brown and C. Creton, Eur. Phys. J. E 9, 35 (2002).

${ }^{18}$ A. Chiche, P. Pareige, and C. Creton, C. R. Acad. Sci., Ser IV: Phys., Astrophys. 1, 1197 (2000).

${ }^{19}$ C. Gay and L. Leibler, Phys. Rev. Lett. 82, 936 (1999); I. Chikina and C. Gay, ibid. 85, 4546 (2000).

${ }^{20}$ B. N. J. Persson, Sliding Friction: Physical Principles and Applications, 2nd ed. (Springer-Verlag, Heidelberg, 2000).

${ }^{21}$ J. H. Dieterich and B. D. Kilgore, PAGEOPH 143, 283 (1994).

${ }^{22}$ B. N. J. Persson, Phys. Rev. B 61, 5949 (2000).

${ }^{23}$ B. N. J. Persson and A. I. Volokitin, Phys. Rev. B 65, 134106 (2002).

${ }^{24}$ Theories of rubber friction for stationary sliding (neglecting the flash temperature effect) have been presented in Ref. 6 and by M. Klüppel and G. Heinrich, Rubber Chem. Technol. 73, 578 (2000).

${ }^{25}$ The kinetic sliding friction depends very weakly on the sliding velocity for low sliding velocities, and extremely low velocities are necessary in order for the friction to be strongly reduced; thus, calculations and experiment show that even sliding velocities as small as $10^{-10} \mathrm{~m} / \mathrm{s}$ may give rise to relatively large kinetic friction.

${ }^{26}$ According to calculations (Ref. 6) (neglecting temperature effects) and measurements [see K. A. Grosch, Proc. R. Soc. London, Ser. A 274, 21 (1963)] (performed at low sliding velocities and different temperatures and then shifted to a common temperature utilizing the frequencytemperature WLF transform), the maximum of $\mu_{k}(v)$ typically occurs at very high velocities, say $1000 \mathrm{~m} / \mathrm{s}$.

${ }^{27}$ A. Galliano, S. Bistac, and J. Schultz, J. Colloid Interface Sci. 265, 372 (2003); A. Galliano, S. Bistac, and J. Schultz, J. Adhes. 79, 973 (2003).

${ }^{28}$ B. N. J. Persson (unpublished).

${ }^{29}$ T. R. Thomas, Rough Surfaces, 2nd ed. (Imperial College Press, London, 1999).

${ }^{30}$ D. Stauffer and A. Aharony, Percolations Theory (VCH, Weinheim, 995).

${ }^{31}$ K. N. G. Fuller and D. Tabor, Proc. R. Soc. London, Ser. A 345, 327 (1975); G. A. D. Briggs and B. J. Briscoe, J. Phys. D 10, 2453 (1977); K. N. G. Fuller and A. D. Roberts, ibid. 14, 221 (1981); K. Kendall, ibid. 4, 1186 (1971); 6, 1782 (1973); 8, 115 (1975); See also the beautiful review article of K. Kendall, Contemp. Phys. 21, 277 (1980).

${ }^{32}$ K. L. Johnson, K. Kendall, and A. D. Roberts, Proc. R. Soc. London, Ser. A 324, 301 (1971). 